University of Nebraska - Lincoln

DigitalCommons@University of Nebraska - Lincoln

2008

\title{
Vortex Ferroelectric Domains
}

Alexei Gruverman

University of Nebraska-Lincoln, agruverman2@unl.edu

D. $\mathrm{Wu}$

North Carolina State University, Raleigh, NC

H.-J. Fan

University of Cambridge, Cambridge CB2 3EQ, UK

I. Vrejoiu

Max Planck Institute of Microstructure Physics, Weinberg 2, D-06120 Halle/Saale, Germany

M. Alexe

Max Planck Institute of Microstructure Physics, Weinberg 2, D-06120 Halle/Saale, Germany

See next page for additional authors

Follow this and additional works at: https://digitalcommons.unl.edu/physicsgruverman

Part of the Physics Commons

Gruverman, Alexei; Wu, D.; Fan, H.-J.; Vrejoiu, I.; Alexe, M.; Harrison, R. J.; and Scott, J. F., "Vortex Ferroelectric Domains" (2008). Alexei Gruverman Publications. 49.

https://digitalcommons.unl.edu/physicsgruverman/49

This Article is brought to you for free and open access by the Research Papers in Physics and Astronomy at DigitalCommons@University of Nebraska - Lincoln. It has been accepted for inclusion in Alexei Gruverman Publications by an authorized administrator of DigitalCommons@University of Nebraska - Lincoln. 


\section{Authors}

Alexei Gruverman, D. Wu, H.-J. Fan, I. Vrejoiu, M. Alexe, R. J. Harrison, and J. F. Scott 
Published in Journal of Physics: Condensed Matter 20 (2008) 342201 (5pp); doi:10.1088/0953-8984/20/34/342201 Copyright @ 2008

IOP Publishing Ltd. Used by permission. Online at http://stacks.iop.org/JPhysCM/20/342201

Submitted July 16, 2008; published August 1, 2008.

FAST TRACK COMMUNICATION

\title{
Vortex ferroelectric domains
}

\author{
A. Gruverman, ${ }^{1}$ D. Wu, ${ }^{2}$ H-J. Fan, ${ }^{3}$ I. Vrejoiu, ${ }^{4}$ M. Alexe, ${ }^{4}$ R. J. Harrison, ${ }^{3}$ and J. F. Scott ${ }^{3}$ \\ ${ }^{1}$ Department of Physics and Astronomy, University of Nebraska-Lincoln, Lincoln, NE 68588-0111, USA \\ ${ }^{2}$ Department of Physics, North Carolina State University, Raleigh, NC 27695, USA \\ ${ }^{3}$ Earth Sciences Department, University of Cambridge, Cambridge CB2 3EQ, UK \\ ${ }^{4}$ Max Planck Institute of Microstructure Physics, Weinberg 2, D-06120 Halle/Saale, Germany
}

\begin{abstract}
We show experimental switching data on microscale capacitors of lead-zirconate-titanate (PZT), which reveal time-resolved domain behavior during switching on a $100 \mathrm{~ns}$ scale. For small circular capacitors, an unswitched domain remains in the center while complete switching is observed in square capacitors. The observed effect is attributed to the formation of a vortex domain during polarization switching in small circular capacitors. This dynamical behavior is modeled using the Landau-Lifshitz-Gilbert equations and found to be in agreement with experiment. This simulation implies rotational motion of polarization in the xy plane, a Heisenberg-like result supported by the recent model of Naumov and Fu (2007 Phys. Rev. Lett. 98 077603), although not directly measurable by the present quasi-static measurements.
\end{abstract}

Over the past 60 years several physicists have considered the possibility that magnetic spins or electric polarization vectors might order not rectilinearly but in circles or toroids. These vortex domain structures (sometimes called "closure domains") in ferroic materials were first predicted by Landau and Lifshitz in 1935 [1] and by Kittel in 1946 [2], who showed that formation of circular domains was likely in ferromagnetic nanodots due to the surface boundary conditions. In 1979, Mermin [3] analyzed their two-dimensional structures in terms of winding numbers, which was extended by Muxworthy et al. [4] to three-dimensional "vortex" domains. Such domain patterns are well known in nanoferromagnets, such as Fe/Ti ilmenite, and are best studied by electron holography [5], but have not been reported in ferroelectrics experimentally, although predicted very recently [6,7]. The extension to ferroelectrics was made around 1984 by Ginzburg et al. [8] and was recently further developed using ab initio calculations by Naumov et al. [9]. We note that toroidal domains can arise for two unrelated physical reasons: (1) finite size effects and boundary conditions (present work) and (2) magnetoelectric coupling in multiferroic materials [7, 8, 10-12]. The present work does not involve magnetism.

Here we report the direct observation of a doughnutshape domain pattern appearing during polarization reversal in micrometer-sized ferroelectric capacitors [13]. The switching behavior of the out-of-plane polarization component $P_{z}$ showing nucleation and wall propagation along the electrode edge, as well as evolution of the in-plane polarization components $P_{x}$ and $P_{y}$, were simulated using the Landau-Lifshitz-Gilbert (LLG) equations. We show that the LLG model completely reproduces the $P_{z}$ behavior, implying that the $P_{x}$ and $P_{y}$ switching kinetics are also adequately modeled. Detailed analysis of the simulated inplane polarization pattern indicates the formation of the transient vortex domain during polarization reversal.

The switching experiments were carried out via ultrafast stroboscopic piezoresponse force microscopy (PFM) where a conducting probing tip in contact with the top electrode was used for application of an electrical bias and detection of the domain patterns in the capacitors. A ferroelectric layer underneath the top electrode was partially switched by applying a short (less than total switching time) voltage pulse with amplitude above the threshold value. Then the entire capacitor was scanned in the PFM mode to visualize the resulting distribution of the out-ofplane, or vertical, $P_{z}$ polarization component developed in the capacitor. The field was then applied for a longer time interval and the imaging PFM procedure repeated. Because domain nucleation is nearly $100 \%$ inhomogeneous in PZT, occurring at the same defect sites, it was then possible to superimpose the collection of images obtained for differ- 

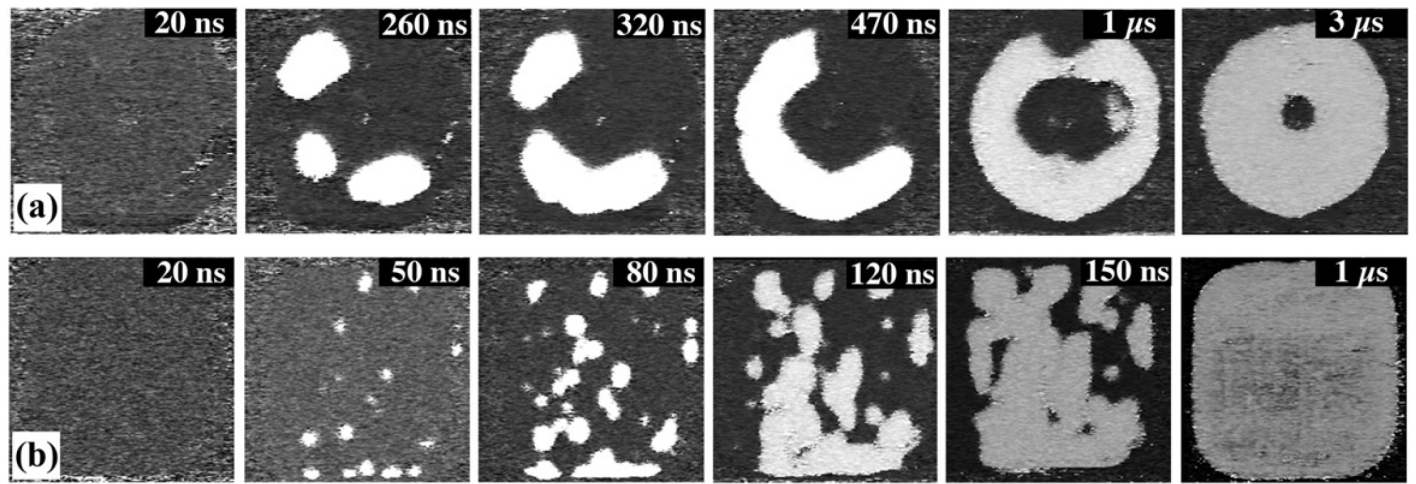

Figure 1. PFM images of instantaneous domain configurations with out-of-plane polarization component $P_{z}$ developing at different stages of polarization reversal in (a) 1- $\mu \mathrm{m}$-diameter circular capacitor and (b) $2.5 \mu \mathrm{m}$ edge square capacitor. Applied electric field is $700 \mathrm{kV} \mathrm{cm}^{-1}$. The total switching time for the domain in the center of the disc is about $1 \mathrm{~s}$.

ent pulse durations to form a slow-motion video of nucleation and domain wall motion during polarization reversal [13]. Domain imaging has been performed through the top electrode by applying an oscillating bias of $0.6 \mathrm{~V}$ (peakto-peak) at $10 \mathrm{kHz}$. Samples used in this study were $50 \mathrm{~nm}$ thick epitaxial (001)-oriented $\mathrm{PbZr}_{0.2} \mathrm{Ti}_{0.8} \mathrm{O}_{3}$ (PZT 20/80) capacitors with circular $1-\mu \mathrm{m}$ - diameter and square $2.5 \mu \mathrm{m}$ edge Pt top electrodes fabricated by e-beam lithography and lift-off. PZT layers were fabricated by pulsed laser deposition on $\mathrm{SrRuO}_{3} / \mathrm{SrTiO}_{3}(001)$ substrates. Details on film fabrication and basic properties are given elsewhere [14].

The snapshots of instantaneous domain patterns corresponding to the vertical component of polarization $P_{z}$ developing in circular and square PZT capacitors during polarization reversal are shown in Figure 1. It can be seen that in the circular capacitor domains nucleate at the electrode edge and propagate around the circumference forming a doughnut-type structure (with an inner circular region having polarization $P_{z}$ pointing down and the outer ring region being oriented upward) in just a few microseconds (Figure 1(a)). This structure remains for $>1 \mathrm{~s}$, after which the unswitched central domain collapses to leave a uniformly polarized ground state. In contrast, the squareshaped capacitors never exhibit a doughnut-type domain structure (Figure 1(b)). Instead, switching proceeds via randomly distributed nucleation events with subsequent isotropic lateral domain growth. In general, the observed features of domain kinetics can be summarized as follows.

(1) The doughnut-type domains occur only in small circular capacitors $(<1 \mu \mathrm{m}$ in diameter) and never in larger square- shaped capacitors.

(2) There are always several nucleation sites and all the nucleation occurs near the walls, but none occur at the domain walls.

(3) The doughnut-type domain structure develops within the time period of several microseconds, lasts for several seconds and then transforms to a single domain state.

The experimentally observed features of domain kinetics in circular capacitors lead us to assume that it might be not simply due to the flipping of polarization but is actually a result of vortex state formation during switching. Vortex domains are well known in magnetism. Three-dimensional micromagnetic modeling by Muxworthy et al. [4] predicted that magnetostatic interactions can generate vortex states in submicrometer-sized grains which strongly depend on the grain size and anisotropy. The nucleation and evolution of these vortex domain states is unclear due to periodic boundary conditions imposed in the modeling, which obscures the inhomogeneous surface nucleation process in real materials. Note that, in ferroelectrics, polarization does not generally rotate continuously in space, so that the structures shown in [4] with magnetization vectors curling around a central core are only qualitative in the description of ferroelectrics. The ferroelectric case can be approximated in magnetic models by letting the anisotropy/exchange ratio become very large. The metastable nature of the vortex domain state was not emphasized by Muxworthy et al. [4] but is probably another reason why vortex domains have not been reported before in ferroelectrics: they are only metastable, lasting a few seconds. The collapse of the vortex state follows from Maxwell's equations, particularly

$$
-\nabla \times D=\mathrm{d} B / \mathrm{d} t
$$

which implies that any vortex is unstable against decay unless $-\nabla \times D=0$.

The simulation of ferroelectric vortex domain formation and switching has been performed using the LLG User Manual [15], which in turn is based upon the micromagnetic equations of motion for a Heisenberg magnet as developed by Scheinfein et al. [16]. The time evolution of a magnetization configuration described by the Landau-Lifshitz-Gilbert equation has been solved with the pseudo-spin configuration relaxed iteratively. For the application to ferroelectric PZT we adjusted the pseudo-spin parameters such that the anisotropy is larger than in ferromagnets and the exchange energy is smaller. The LLG simulation allowed us to reproduce all the details of the experimental data on domain switching (Figure 2).

(1) The doughnut domains occur only in small circularshaped and never in square-shaped capacitors.

(2) There are always several nucleation sites. 

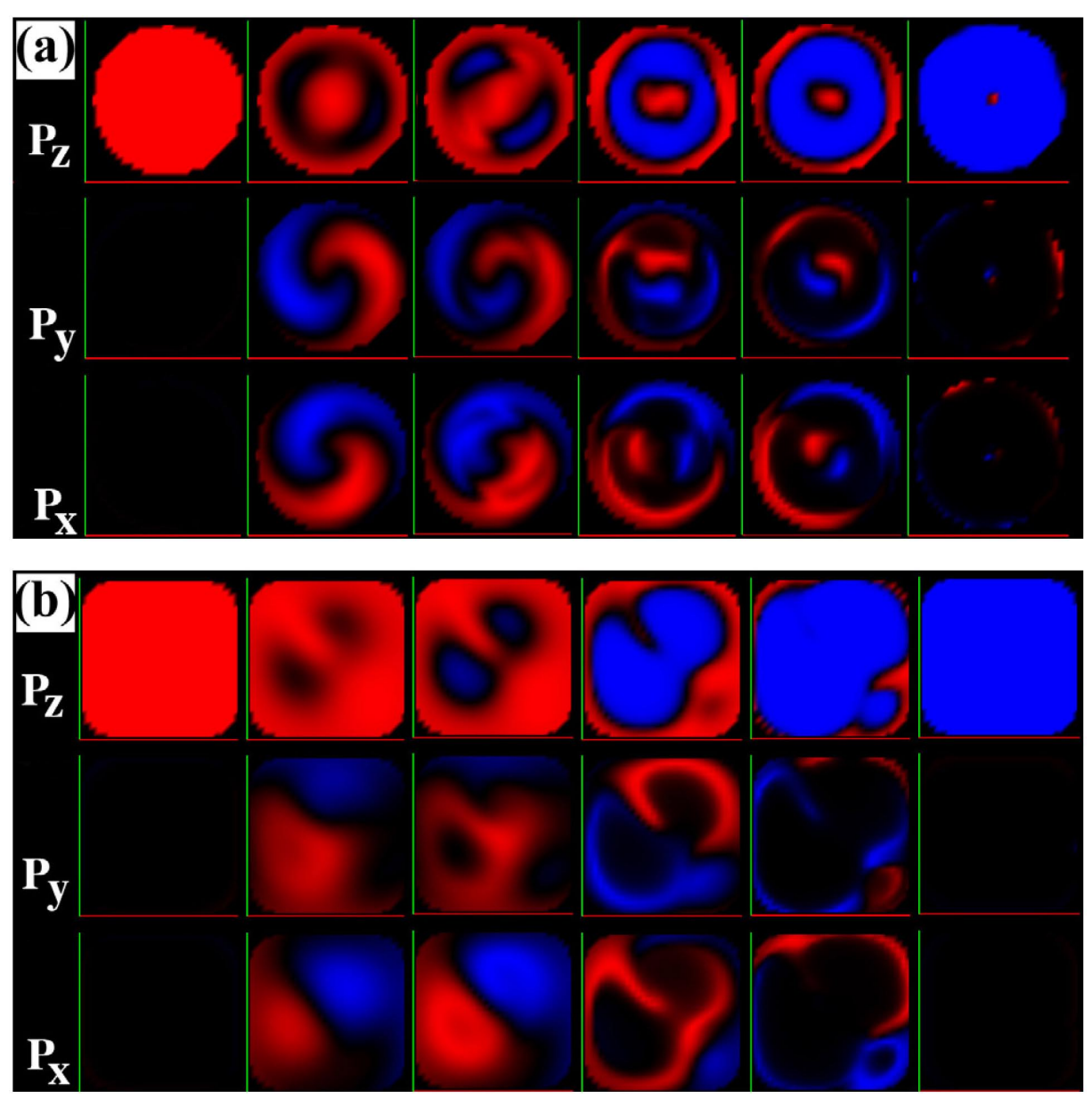

Figure 2. Simulation of domain switching kinetics in: (a) 1- $\mu \mathrm{m}$-diameter circular capacitor and (b) $2.5 \mu \mathrm{m}$ edge square capacitor. The top trace shows the time evolution of out-of-plane polarization component $P_{z}$; lower traces illustrate time evolution of inplane polarization components $P_{x}$ and $P_{y}$, which cannot be measured with the present PFM technique. Red corresponds to positive polarization directions: $P_{z}$ - out-of-plane, $P_{y}$ - upward, $P_{x}-$ to the right; blue indicates negative directions.
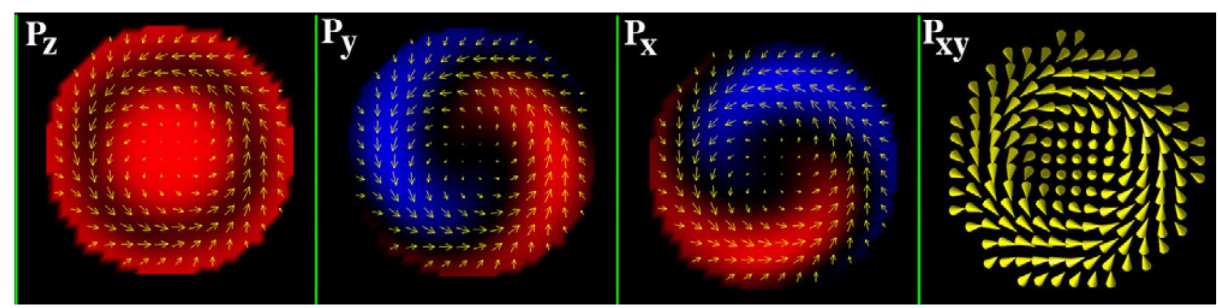

Figure 3. Two-dimensional maps of polarization components $P_{z}, P_{y}$, and $P_{x}$ and in-plane polarization $P_{x y}$ corresponding to an instantaneous domain pattern in the $1-\mu \mathrm{m}$-diameter circular capacitor during switching; the in-plane polarization map $P_{x y}$ illustrates a vortex structure similar to the one in [9].

(3) All the nucleation occurs near the circular capacitor edge, but none occur at the edge.

(4) The switching occurs in approximately 100 time steps and the doughnut-type center remains for approximately $5 \times 10^{6}$ time steps and then it vanishes. Our time steps thus each represent $\sim 200 \mathrm{~ns}$ in the experiments.

One obvious limitation of the present PFM experiments and modeling should be mentioned here. In the LLG simu- lation, we watch the actual dynamical switching process in detail, while the domain walls are moving. This process involves not only a simple Ising-like up and down flipping of polarization but also a development of the in-plane component of polarization, which arises prior to the complete polarization reversal. However, it is difficult to verify this via direct PFM experiment for at least two reasons. First, the actual domain switching measurements using stroboscopic PFM are quasistatic. The PZT capacitors are par- 
tially switched and allowed to remain in this state for a time required for PFM domain image acquisition, which is much longer than the switching time. On the other hand, the vortex-type domains represent non-steady polarization state with the in-plane components appearing only during dynamic switching. Second, during PFM measurements through the top electrodes, the lateral PFM signal is inevitably affected by the contribution of $d_{31}$ and $d_{32}$ constants of the piezoelectric tensor of tetragonal PZT, which makes unambiguous detection of the in-plane polarization difficult [17].

The line of inference is that, since the numerical simulation very closely replicates experimental evolution of the $P_{z}$ polarization as a function of time, the evolution of polarization components $P_{x}$ and $P_{y}$ obtained in the numerical simulation should also adequately reflect the actual kinetics of the in-plane polarization during switching. In Figure 3, a two-dimensional map of the in-plane polarization distribution $P_{x y}$, obtained by combining the simulated instantaneous maps of $P_{x}$ and $P_{y}$ components, shows the vortex structure consistent with the pattern modeled by Naumov and $\mathrm{Fu}$ [7] and with recent results by Junquera [18].

A simpler but analogous description for the domain patterns observed in Figures 1 and 2 can be done by means of Bessel functions, which are used for description of the propagation of electric currents in cylindrical waveguides [19-21]. Let us consider the circular PZT capacitors to be cylindrical waveguides. In this case, the lower TEM modes are well known and they look like the patterns we see. So we might treat the domain wall nucleation and growth in PZT capacitors as occurring at the high-field regions of a circular waveguide of radius $B$. The doughnut-shape domain can be described by an internal radius $a$ and an external radius $b$, with $b<B$. One finds that the impedance $Z$ of the transmission "line" is the ratio of voltage between the inside and outside to the axial current flowing normal to the disc. In our case the axial current $J$ is not ohmic but instead a displacement current, so borrowing the waveguide results [22] we can write

$$
\mathrm{d} P / \mathrm{d} t=J=(2 n / c) V(\mu / \varepsilon)^{1 / 2} \ln (b / a)
$$

where $\varepsilon$ is the dc current $\varepsilon(0)$ and not $\varepsilon(\infty)=n^{2}$. Although this approach is only qualitative (it yields only order-ofmagnitude agreement with the measured values of $\mathrm{d} P / \mathrm{d} t$ ), the idea of replacing real current $J(t)$ with displacement current $i(t)=\mathrm{d} P / \mathrm{d} t$ in ferroelectric nanorods during switching merits further consideration.

Figure 2 shows both radial and bending mode Bessel function distribution of polarization. To understand the dynamic response of $P_{x}$ and $P_{y}$ components, one must Fouriertransform the spatially uniform applied field $E(t)$ to $E(f)$. A pulsed field has two main Fourier components: $f_{1}$ at the reciprocal of the rise time and $f_{2}$ at the reciprocal of the pulse width. Using the known values of Young's modulus $Y$, density $\rho$, and Poisson's ratio $v$, the lowest radial frequency for a thin PZT disc of thickness $d=50 \mathrm{~nm}$ can be estimated as

$$
f_{1}=(2.09 / 2 \pi B)\left(Y /\left(\rho\left(1-v^{2}\right)\right)^{1 / 2}=218 \mathrm{MHz}\right.
$$

and the next-lowest bending mode as

$$
f_{2}=\left(38.5 d / 2 \pi B^{2}\right)\left(Y /\left(12 \rho\left(1-v^{2}\right)\right)^{1 / 2}=13.2 \mathrm{MHz} .\right.
$$

Hence, applied voltage pulses with a $\tau_{\mathrm{r}}=5 \mathrm{~ns}$ rise time will resonate strongly with the radial Bessel function at approximately $200 \mathrm{MHz}$ and the $100 \mathrm{~ns}$ wide pulses will resonate strongly with the bending mode. Hence, in our experiment, both bending and radial modes are resonantly driven, suggesting a possibility of moving $90^{\circ}$ domains. This also explains why such vortex domains were never observed with slow rise time or sinusoidal voltages. It is worthwhile emphasizing that similar vortex domain structures in ferroelectric cylinders were recently predicted in Luk'yanchuk's theoretical paper [23], which shows analytically that the stable domain configuration in a thin PZT disc under an applied field is a second-order Bessel function appearing with an unswitched edge and a "hole" in the middle.

In summary, we report observation of the instantaneous $P_{z}$ domain configurations during switching in micron-sized ferroelectric capacitors on a 100 ns timescale. Formation of the doughnut-shape $P_{z}$ domain pattern with an unswitched circular domain at its center is attributed to a vortex domain structure developing during dynamic switching. This conclusion is made based on numerical switching simulations using the Landau-Lifshitz-Gilbert equations which agree very closely with experimental data for both circular and square-shaped capacitors. The simulations predict a significant in-plane polarization during switching in circular capacitors, which agrees with the model of Naumov and $\mathrm{Fu}[7]$ but is beyond present experimental detection.

\section{References}

[1] Landau L D and Lifshitz E M 1935 On the theory of the dispersion of magnetic permeability in ferromagnetic bodies Phys. J. Sowjetunion 8153

[2] Kittel C 1946 Theory of the structure of ferromagnetic domains in films and small particles Phys. Rev. 70965

[3] Mermin N D 1979 The topological theory of defects in ordered media Rev. Mod. Phys. 51591

[4] Muxworthy A R, Dunlop D J, and Williams W 2003 Hightemperature magnetic stability of small magnetite particles J. Geophys. Res. 108 2281; Williams W and Wright T M 1998 High-resolution micromagnetic models of fine grains of magnetite J. Geophys. Res. 10330537

[5] Harrison R J, Dunin-Borkowski R E, and Putnis A 2002 Direct imaging of nanoscale magnetic interactions in minerals Proc. Natl Acad. Sci. USA 9916556

[6] Wu Z Q et al. 2007 Unusual vortex structure in ultrathin $\mathrm{Pb}\left(\mathrm{Zr}_{0.5} \mathrm{Ti}_{0.5}\right) \mathrm{O}_{3}$ films J. Appl. Phys. 101014112

[7] Naumov I I and Fu H X 2007 Vortex-to-polarization phase transformation path in ferroelectric $\mathrm{Pb}(\mathrm{ZrTi}) \mathrm{O}_{3}$ nanoparticles Phys. Rev. Lett. 98077603

[8] Ginzburg V L, Gorbazevitch A A, Kopaev Y V, and Volkov B A 1984 On the problem of superdiamagnetism Solid State Commun. 50 339; Gorbatsevich A A and Kopayev Y V 1994 Toroidal order in crystals Ferroelectrics 161321 
[9] Naumov I I, Bellaiche L, and Fu H X 2004 Unusual phase transitions in ferroelectric nanodisks and nanorods Nature 432737

[10] Scott J F 2005 Ferroelectrics: novel geometric ordering of ferroelectricity Nat. Mater. 413

[11] Van Aken B B, Rivera J P, Schmid H, and Fiebig M 2007 Observation of ferrotoroidic domains Nature 449702

[12] Fiebig M 2005 Revival of magnetoelectric effect J. Phys. D: Appl. Phys. 38 R123

[13] Gruverman A, Rodriguez B J, Dehoff C, Waldrep J D, Kingon A I, Nemanich R J, and Cross J S 2005 Direct studies of domain switching dynamics in thin film ferroelectric capacitors Appl. Phys. Lett. 87082902

[14] Vrejoiu I, Le Rhun G, Pintilie L, Hesse D, Alexe M, and Goesele U 2006 Intrinsic ferroelectric properties of strained tetragonal $\mathrm{PbZr}_{0.2} \mathrm{Ti}_{0.8} \mathrm{O}_{3}$ obtained on layer-bylayer grown, defect-free single-crystalline films Adv. Mater. 181657

[15] Sheinfein M R and Price E A The LLG User Manual Version 2.50; ligmicro@mindspring.com

[16] Scheinfein M R, Unguris J, Blue J L, Coakley K J, Pierce D T, Celotta R J, and Ryan P J 1991 Micromagnetics of domain walls at surfaces Phys. Rev. B 433395
[17] Kalinin S V, Rodriguez B J, Jesse S, Shin J, Baddorf A P, Gupta P, Jain H, Williams D B, and Gruverman A 2006 Vector piezoresponse force microscopy Microsc. Microanal. 12206

[18] Aguado-Puente P and Junquera J 2008 Ferromagnetic-like closure domains in ferroelectric ultrathin films: first-principles simulations Phys. Rev. Lett. 100177601

[19] Sokolnikoff I S and Redheffer R M 1958 Mathematics of Physics and Modern Engineering (New York: McGraw-Hill) p 159

[20] Bakshi U A and Bakshi A V 2006 Transmission Lines and Waveguides (Pune: Technical Publications) chapter 5

[21] Cronin N 1995 Microwave and Optical Waveguides (Bristol: Hilger) chapter 6

[22] Jackson J D 1963 Classical Electrodynamics (New York: Wiley) p 265

[23] Lahoche L, Luk'yanchuk I, and Pascoli G 2008 Stability of vortex phases in ferroelectric easy-plane nano-cylinders http://lanl.arxiv.org/abs/0802.4164 